\title{
A very luminous, highly extinguished, very fast nova - V1721 Aquilae
}

\author{
R. Hounsell ${ }^{1}$, M. J. Darnley ${ }^{1}$, M. F. Bode ${ }^{1}$, D. J. Harman ${ }^{1}$, L. A. Helton ${ }^{2,3}$, and G. J. Schwarz ${ }^{4}$ \\ 1 Astrophysics Research Institute, Liverpool John Moores University, Twelve Quays House, Egerton Wharf, Birkenhead, \\ CH41 1LD, UK \\ e-mail: rah@astro.livjm.ac.uk \\ 2 SOFIA Science Center, USRA, NASA Ames Research Center, M.S. N211-3, Moffett Field, CA 94035, USA \\ 3 Department of Astronomy, The University of Minnesota, 116 Church St. S.E. Minneapolis, MN 55455, USA \\ 4 American Astronomical Society, 2000 Florida Ave., NW Suite 400, Washington, DC 20009-1231, USA
}

Received 5 November 2010 / Accepted 15 March 2011

\begin{abstract}
Studies indicate that fast novae are primarily located within the plane of the Milky Way and slow novae are found within its bulge. Because of high interstellar extinction along the line of sight many novae lying close to the plane are missed and only the brightest seen. One nova lying very close to the Galactic plane is V1721 Aquilae, which was discovered in outburst on 2008 September 22.5 UT. Examination of spectra obtained 2.69 days after outburst revealed very high expansion velocities ( $F W H M$ of the $\mathrm{H} \alpha$ emission $\approx 6450 \mathrm{~km} \mathrm{~s}^{-1}$ ). In this paper we have used available pre- and post-outburst photometry and post-outburst spectroscopy to conclude that the object is a very fast, luminous, and highly extinguished $\left(A_{\mathrm{V}}=11.6 \pm 0.2\right)$ nova system with an average ejection velocity of $\approx 3400 \mathrm{~km} \mathrm{~s}^{-1}$. Pre-outburst near-IR colours from the 2MASS point source catalogue indicate that at quiescence the object is similar to many quiescent classical novae and appears to have a main sequence/sub-giant secondary rather than a giant counterpart. Based on the speed of decline of the nova and its emission line profiles we hypothesise that the axis ratio of the nova ejecta is $\sim 1.4$ and that its inclination is such that the central binary accretion disc is face-on to the observer. As such, the accretion disc's blue contribution to the system's near-IR quiescent colours may be significant. Simple models of the nova ejecta have been constructed using the morphological modelling code XS5, and the results support the above hypothesis. Precise spectral classification of this object has been exceptionally difficult owing to low signal-to-noise levels and high extinction, which has eliminated all evidence of any $\mathrm{He} / \mathrm{N}$ or Fe II emission within the spectra. We suggest two possibilities for the nature of V1721 Aql: that it is a U Sco type RN with a sub-giant secondary or, less likely, that it is a highly energetic bright and fast classical nova with a main sequence secondary. Future monitoring of the object for possible RN episodes may be worthwhile, as would archival searches for previous outbursts.
\end{abstract}

Key words. novae - cataclysmic variables - stars: individual: V1721 Aql

\section{Introduction and observations}

Classical novae $(\mathrm{CNe})$ occur in interacting binary systems consisting of a white dwarf (WD) primary and a main sequence secondary star, which fills its Roche-lobe. They are a subclass of the cataclysmic variables (CVs). Hydrogen-rich material is transferred from the secondary and deposited onto the surface of the WD, usually via an accretion disc (see Bode \& Evans 2008 , for recent review papers). A thermonuclear runaway eventually occurs within the accreted hydrogen-rich layer on the WD surface leading to a nova outburst (see Starrfield et al. 2008). CNe tend to exhibit outburst amplitudes of approximately 10-20 mag (Shara 1981) and eject $10^{-5}-10^{-4} M_{\odot}$ of material at velocities between hundreds to a few thousand $\mathrm{km} \mathrm{s}^{-1}$ (Prialnik \& Kovetz 1995), with outbursts occurring approximately once every $10^{4}-10^{5}$ years. If a nova system is seen to have more than one outburst, it is classed as a recurrent nova $(\mathrm{RN})$. RNe undergo outbursts on a time-scale of decades up to $\sim 100$ years and tend to have higher ejection velocities and lower ejected masses than CNe (Anupama 2008). The secondary star in a RN system is often an evolved star such as a sub-giant or red giant (see also review by Bode 2010).

Novae can be grouped into classes depending on their speed of decline from maximum light ("speed classes",
Payne-Gaposchkin 1957) or the dominance of certain nonBalmer emission lines in their post-outburst spectrum (Williams 1992). These emission lines are either those of $\mathrm{Fe}$ II or $\mathrm{He} / \mathrm{N}$, yielding two spectral classes. The spectra of $\mathrm{He} / \mathrm{N}$ novae tend to exhibit "boxy" structures and high expansion velocities, whereas Fe II spectra are more Gaussian and have lower expansion velocities. Recurrent novae typically show features consistent with the $\mathrm{He} / \mathrm{N}$ novae.

In the Milky Way the distribution of novae shows a strong concentration towards the Galactic plane and the bulge. It has been shown by della Valle et al. (1992) that fast novae are more concentrated toward the Galactic plane $(z<100 \mathrm{pc})$ than slow novae, which are associated with the Galactic bulge extending up to $1 \mathrm{kpc}$. Fast novae are more luminous at peak than slow novae and possess more massive and luminous WDs. Work by Duerbeck (1990) indicates that many of these bright novae, however, go undetected. The reasons for this are high interstellar extinction inherent in the Galactic plane, and that for many novae their speed of decline alone makes them difficult to detect (see Warner 2008; Hounsell et al. 2010, for discussion). Even now, many fast highly extinguished novae are only ever detected and tracked by amateur astronomers.

Nova V1721 Aquilae $\left(\alpha=19^{\mathrm{h}} 06^{\mathrm{m}} 28^{\mathrm{s}} 58, \delta=+07^{\circ} 06^{\prime} 44^{\prime \prime} \cdot 3\right.$; J2000) was discovered on 2008 September 22.5 UT by 
K. Itagkai. The outburst was confirmed on 2008 September 22.586 UT and reached a peak unfiltered magnitude of 14.0. Discovery of the nova was presented in Yamaoka et al. (2008) along with an initial spectral investigation. Post-outburst spectra were obtained on 2008 September 25.19 and 25.25 UT using the Steward Observatory Bok 2.29 m telescope on Kitt Peak via the Boller \& Chivens optical spectrograph (details of the instrumental set-up can be found in Sect. 2.2). Initial analysis of the spectra revealed a broad triple-peaked $\mathrm{H} \alpha$ emission profile with a full width half maximum $(F W H M)$ of $6450 \mathrm{~km} \mathrm{~s}^{-1}$, along with O I $7773 \AA$ and O I $8446 \AA$ structures (Helton et al. 2008). The ejecta velocities derived from the initial analysis of the spectra were very high indeed for a typical $\mathrm{CN}$ and there was initial suspicion that it may be a supernova (S. J. Smartt, priv. comm.). Spectra also indicated that extinction towards the object was high and by comparison to other novae during similar evolutionary phases was estimated to be $A_{\mathrm{V}} \approx 9.3$. Hence, the distance to the nova was initially derived as $5 \mathrm{kpc}$, by assuming at maximum $M_{\mathrm{V}} \approx-9$ (Helton et al. 2008). Due to the faintness of the source at maximum and its rapid decline, follow up spectroscopy of the object has not been possible.

This paper aims to determine the nature of V1721 Aql through the examination of available photometric and spectroscopic data. Archival pre-outburst Two Micron All-Sky Survey ${ }^{1}$ (2MASS; Skrutskie et al. 1995) photometry has enabled the determination of the spectral type of the secondary star within the system. Post-outburst photometric and spectroscopic data have been used to obtain the speed class of the nova, its extinction, the average ejection velocity of the system and potential spectral class. Section 2 presents the analysis of the data; Sect. 3 discusses the results found and the subsequent classification of the object.

\section{Data analysis}

\subsection{Distance determination}

After outburst, V1721 Aql continued to be monitored photometrically until 2008 October 6 UT; these results are reproduced in Fig. 1 . These data indicate that $t_{2} \approx 6$ days for the nova, classifying it as very fast (Payne-Gaposchkin 1957). Using these data and the maximum magnitude-rate of decline relation (MMRD, McLaughlin 1945) with parameters from Downes \& Duerbeck (2000), we derive an absolute magnitude $M_{\mathrm{V}}=-9.4 \pm 0.5$.

The extinction towards V1721 Aql is thought to be extremely high, with as noted above, an estimate of $A_{\mathrm{V}} \approx 9.3$ given in Helton et al. (2008). This however, is based purely upon comparisons with other novae at a similar early evolutionary state. In order to obtain an independent extinction we made use of the Rowles \& Froebrich (2009) extinction maps which have a high spatial resolution and are able to detect a greater number of small-scale high extinction cores compared to other maps. These extinction maps are generated using 100 nearest neighbour stars and give an $A_{\mathrm{V}}=11.6 \pm 0.2$, much higher than the original estimate. Using the more accurate extinction from Rowles \& Froebrich (2009), we derive a distance to V1721 Aql of $2.2 \pm 0.6 \mathrm{kpc}$.

The Galactic coordinates of the object are $l^{\mathrm{II}}=41^{\circ}, b^{\mathrm{II}}=$ $-0.1^{\circ}$. This indicates that nova V1721 Aql is located very close

\footnotetext{
1 The Two Micron All Sky Survey, is a joint project of the University of Massachusetts and the Infra-red Processing and Analysis Centre California Institute of Technology, funded by the National Aeronautics and Space Administration and the National Science Foundation.
}

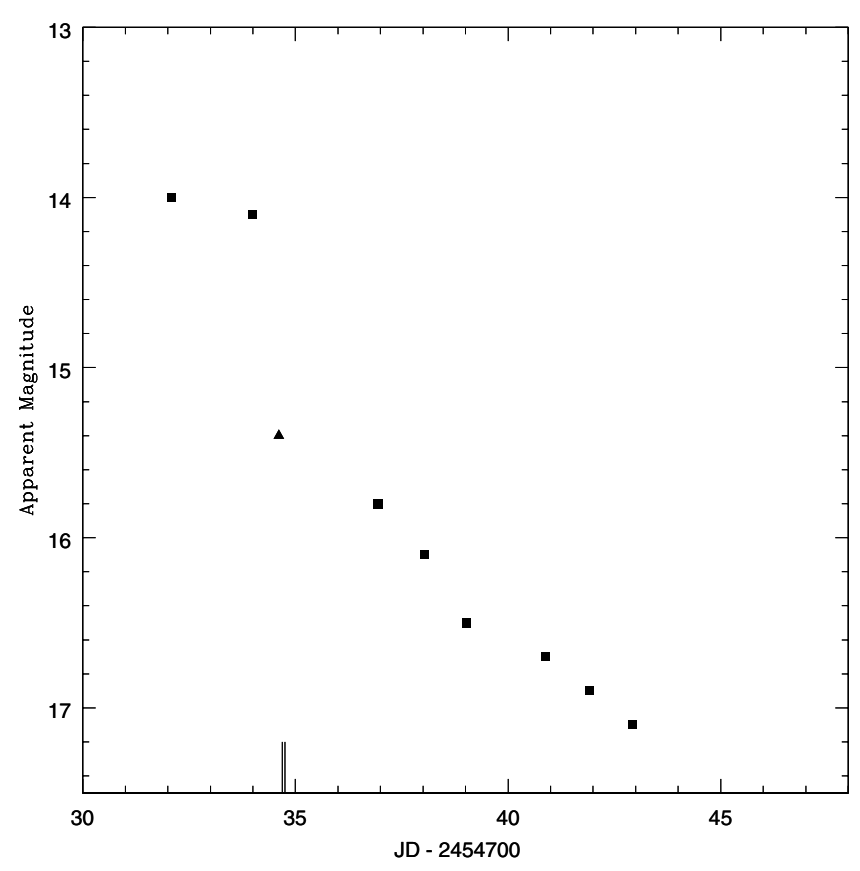

Fig. 1. Apparent magnitudes of Nova V1721 Aql as observed by K. Itagaki (squares - unfiltered) and R. King (triangles - Visual). These results are from VSNET $^{2}$ and $\mathrm{AAVSO}^{3}$. The two additional tick marks on the $x$-axis represent the dates on which the blue (see Fig. 2) and red (see Fig. 3) spectra were taken.

to the Galactic plane with $z=2.5 \mathrm{pc}$, and is in a region of the sky in which it is typically very difficult to observe novae because of high extinction along the line of sight.

\subsection{Post-outburst spectra}

Post-outburst spectra were obtained on 2008 September 25.19 and 25.25 UT using the Steward Observatory Bok $2.29 \mathrm{~m}$ telescope on Kitt Peak with the Boller \& Chivens optical spectrograph, and are presented in Figs. 2 and 3. The "Blue" set-up utilised a $4001 \mathrm{~mm}^{-1} 1$ st order grating with a UV blocking filter to prevent order contamination below $\sim 3600 \AA$. The spectral coverage was from $\sim 3600$ to $\sim 6750 \AA$ at a spectral resolution of roughly $2.8 \AA$ pixel $^{-1}$. The "Red" set-up was identical but with the grating centred near $7600 \AA$ providing coverage from $\sim 6000$ to $\sim 9250 \AA$ and with a blocking filter effective below $4800 \AA$. Flat fielding was performed using a continuum arc lamp. Red observations at wavelengths beyond $\sim 7700 \AA$ are subject to fringing effects arising at the CCD that are unable to be corrected by flat fielding. The effect of this fringing on the data depends upon the target position on the sky and the target intensity. Wavelength calibration was performed using $\mathrm{He}-\mathrm{Ar}-\mathrm{Ne}$ calibration lamps at each target position. The spectroscopic standard Wolf 1346 was used for flux calibration. Spectra have also been corrected for heliocentric velocity and reddening $\left(A_{\mathrm{V}}=11.6\right)$. All data reduction was performed in $\mathrm{IRAF}^{4}$ following standard optical data reduction procedures.

\footnotetext{
2 Available from http://ooruri.kusastro.kyoto-u.ac.jp

3 Available from http://www. aavso.org

${ }^{4}$ IRAF is distributed by the National Optical Astronomy Observatory, which is operated by the Association of Universities for Research in Astronomy (AURA) under cooperative agreement with the National Science Foundation.
} 


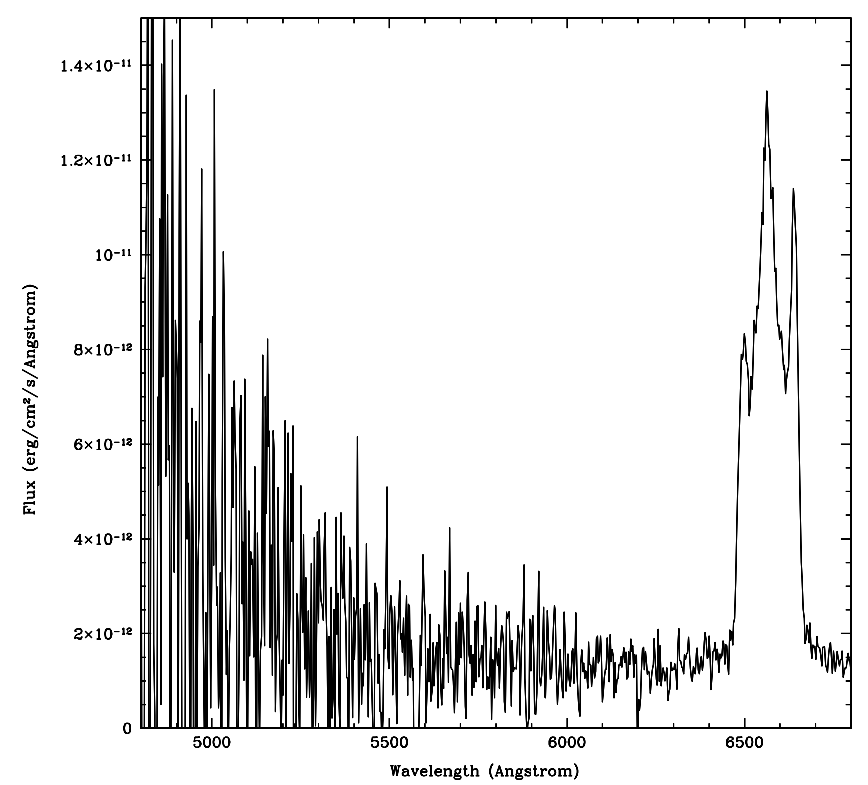

Fig. 2. Heliocentric velocity and extinction corrected $\left(A_{\mathrm{V}}=11.6\right.$, see Sect. 2.1) optical spectrum of V1721 Aql, taken on 2008 September 25.19 (2.69 days after discovery) with the Steward Observatory Bok $2.29 \mathrm{~m}$ telescope.

The Blue spectrum of V1721 Aql is presented in Fig. 2. It is important to note that this spectrum is devoid of detectable emission lines blue-wards of $\mathrm{H} \alpha$, likely owing to the very high extinction. Because of the absence of $\mathrm{H} \beta$ emission in the spectrum, a lower limit on the extinction is obtained using the Balmer decrement for Case B HI recombination and the observed intensity ratio of $\mathrm{H} \alpha$ and $\mathrm{H} \beta$. We note that this spectrum is taken early in the nova outburst and although the nova is very fast, conditions may not yet be those of Case B. From this we estimate a lower limit of $A_{\mathrm{V}} \geq 8$. This value is consistent with both the above determinations of $A_{\mathrm{V}}$ and helps to confirm that the extinction is indeed high.

The Red spectrum of V1721 Aql is shown in Fig. 3 and indicates the presence of a triple-peaked $\mathrm{H} \alpha$ emission line along with emission structures corresponding to O I $7773 \AA$ A and O I $8446 \AA$. It is necessary to determine if the "boxy" structure around $\mathrm{H} \alpha$ consists of purely $\mathrm{H} \alpha$ or combined lines of $\mathrm{H} \alpha+[\mathrm{N} \mathrm{II}] 6482$, $6548,6584,6611 \AA$ A. However, although [N II] is expected in the spectra, this early in the outburst the [N II] line strength is unlikely to be significant in comparison to $\mathrm{H} \alpha$ and so an unlikely contributor to the boxy structure. An absence of [N II] altogether may simply be owing to the fact that the nova has indeed been caught at maxima and the lines tend to develop a little later in the outburst. For example the fast, potentially U Scolike nova V2491 Cyg, showed evidence of [N II] 4.62 days after peak magnitude, with these lines becoming more defined 32.7 to 108 days after peak (Munari et al. 2011b). The lack of [N II] $5755 \AA$ at shorter wavelengths also contradicts the idea of a strong N II presence, although this line may have been missed due to a combination of the object's rapid evolution and high extinction. Additionally, the observed emission peaks at the blue and red edge of the $\mathrm{H} \alpha$ profile in V1721 Aql are nearly symmetric and expected positions of potential [N II] contaminants are not.

A relative velocity diagram of the $\mathrm{H} \alpha$, O I $7773 \AA$, and O I $8446 \AA$ structures is given in Fig. 4. This diagram indicates that the $\mathrm{H} \alpha$ and $\mathrm{O}$ I lines contain similar weak blue/strong red

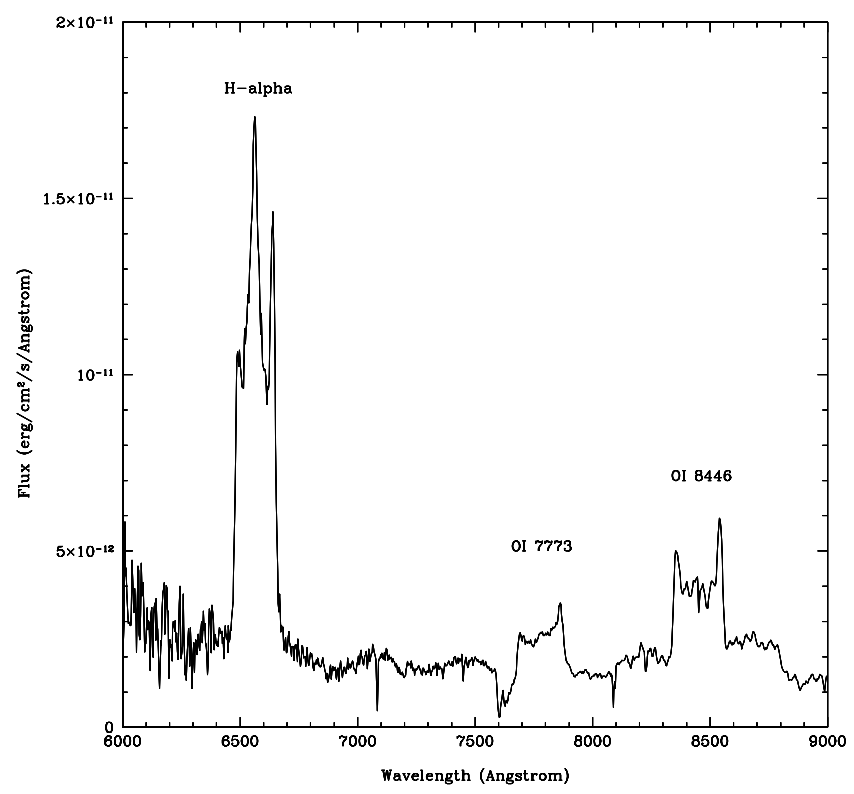

Fig. 3. Heliocentric velocity and extinction corrected $\left(A_{\mathrm{V}}=11.6\right.$, see Sect. 2.1) optical spectrum of V1721 Aql, taken on 2008 September 25.25 (2.75 days after discovery) with the Steward Observatory Bok $2.29 \mathrm{~m}$ telescope.

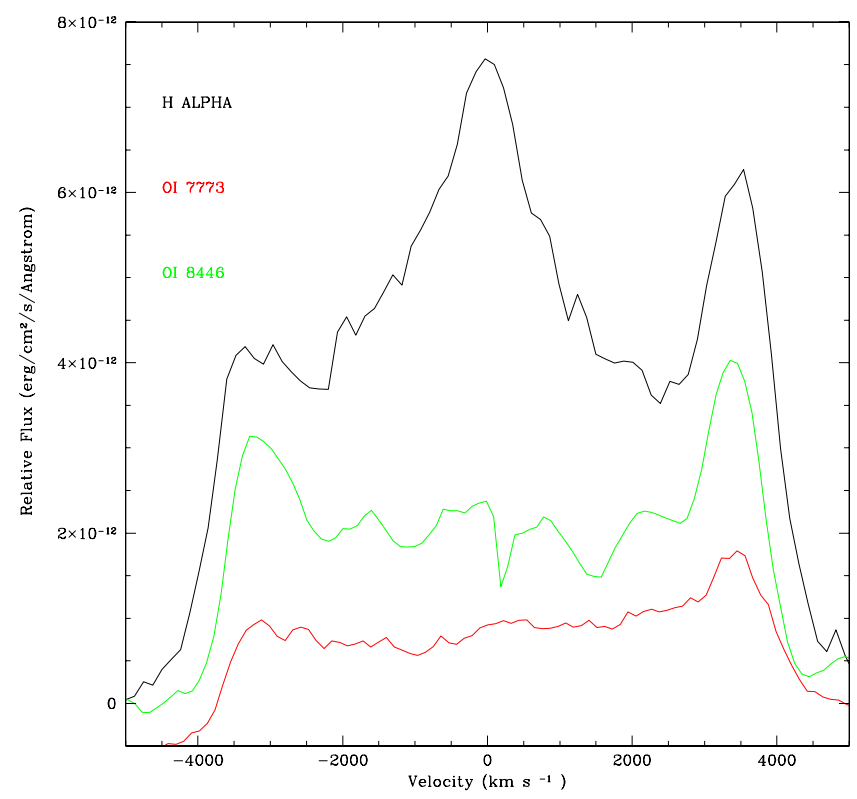

Fig. 4. Relative velocity diagram of the H $\alpha$, O I $7773 \AA$, and O I $8446 \AA$ structures. Note that lines have been off-set on the $y$-axis for ease of comparison.

wing morphologies, however, the $\mathrm{H} \alpha$ central peak is much more prominent and may arise in an emitting region distinct from the other components of the emission profile. The velocity shifts of the three components are also similar which supports the hypothesis that the $\mathrm{H} \alpha$ structure consists of $\mathrm{H} \alpha$ emission only.

In order to identify any potential emission lines that may be contaminating the $\mathrm{H} \alpha$ structure a spectral fit of the region (using the Red spectrum, Fig. 3) was conducted using STSDAS's 5 Specfit, the results of which are presented in Fig. 5 and Table 1. It should be noted that the central $\mathrm{H} \alpha$ peak was fit by two

5 STSDAS is a product of the Space Telescope Science Institute, which is operated by AURA for NASA. 
Table 1. Wavelength, FWHM, and relative velocity of fitted components of the triple-peaked H $\alpha$ structure presented in Fig. 5.

\begin{tabular}{cccc}
\hline \hline Gaussian & Wavelength $(\AA)$ & $F W H M\left(\mathrm{~km} \mathrm{~s}^{-1}\right)$ & Relative velocity $\left(\mathrm{km} \mathrm{s}^{-1}\right)$ \\
\hline 1 & $6493 \pm 3$ & $1400 \pm 100$ & $-3200 \pm 100$ \\
2 & $6563 \pm 1$ & $4300 \pm 100$ & $-10 \pm 50$ \\
3 & $6563 \pm 1$ & $800 \pm 100$ & $-10 \pm 50$ \\
4 & $6639.6 \pm 0.4$ & $1260 \pm 50$ & $3510 \pm 20$ \\
\hline
\end{tabular}

Table 2. Wavelength, FWHM, and relative velocity of primary fitted components of the triple peaked O I 8446 A structure presented in Fig. 6 .

\begin{tabular}{cccc}
\hline \hline Gaussian & Wavelength $(\AA)$ & $F W H M\left(\mathrm{~km} \mathrm{~s}^{-1}\right)$ & Relative velocity $\left(\mathrm{km} \mathrm{s}^{-1}\right)$ \\
\hline 1 & $8358.7 \pm 0.5$ & $1110 \pm 40$ & $-3100 \pm 20$ \\
3 & $8447 \pm 1$ & $3200 \pm 200$ & $40 \pm 50$ \\
6 & $8541.9 \pm 0.4$ & $930 \pm 30$ & $3410 \pm 10$ \\
\hline
\end{tabular}

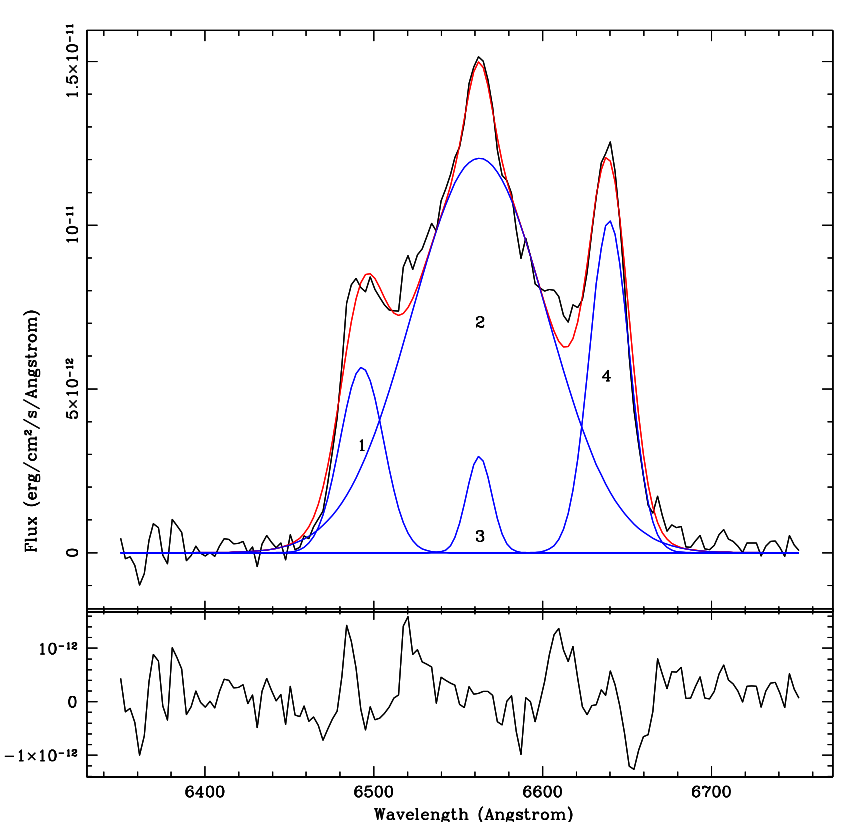

Fig. 5. Observed $\mathrm{H} \alpha$ structure (black line) with the sum of Specfit Gaussian components (red line). The blue lines represent separate Gaussian components. See Sect. 3 for further discussion. The lower plot shows the residual to the fit.

separate Gaussians with the second component added as a correction to the first in compensation for the oversimplification of the fit, a possible physical explanation for this is given in Sect. 3 . The O I $8446 \AA$ structure has also been fit with these results presented in Fig. 6 and Table 2. It is evident that fringing occurs within the spectra at wavelengths $\gtrsim 8000 \AA$. The effect of this fringing has been to contribute to components 2 and 5 of Fig. 6 and to create fine structure long-ward of the O I $8446 \AA$ profile. It was therefore necessary to fit these contaminating structures which would otherwise interfere with the results. Taking fringing effects into account the central structure of the O I $8446 \AA$ line profile is most likely relatively flat. Although there are slight inherent differences in the strengths of the red/blue peaks in the profiles of the O I 7773 and $8446 \AA$ features, the intrinsic shape before fringing effects of the $8446 \AA$ feature is likely very similar to the $7773 \AA$ feature, as illustrated in Fig. 4. The fitting of the O I $7773 \AA$ profile has not been conducted as this structure has been severely truncated on the blue edge by an atmospheric absorption feature.

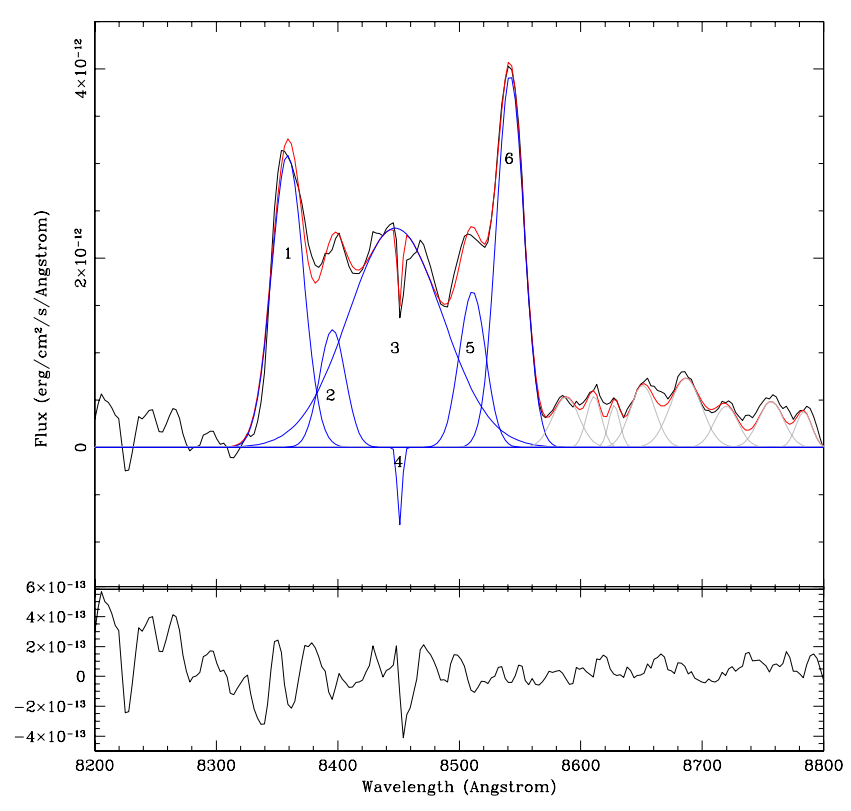

Fig. 6. Observed O I $8446 \AA$ structure (black line) with the sum of Specfit Gaussian components (red line). The blue lines represent separate Gaussian components. Gaussian 4 represents a spectral artifact. Gaussians 2 and 5 represent components within the profile that are partially caused by fringing. Grey Gaussians represent fine structure caused by fringing effects. All fringing effects required fitting in order to produce the best overall match with observations. The lower plot shows the residual to the fit.

We find that no other spectral lines expected to be found in novae match the wavelengths presented within Tables 1 and 2 . Given this, and that the blue and red wings of the $\mathrm{H} \alpha$, O I 7773, and O I 8446 profiles are similar, we conclude that these structures consist of $\mathrm{H} \alpha$ and $\mathrm{O}$ I only. Combining the relative velocities of $\mathrm{H} \alpha$ Gaussians 1 and 4 gives a mean expansion velocity of $V_{\exp }=3400 \pm 200 \mathrm{~km} \mathrm{~s}^{-1}$. The structure of each line profile may also tell us something about the nova ejecta geometry (see Sect. 2.4 for further discussion).

On examination of both blue and red spectra, no evidence of $\mathrm{Fe} \mathrm{II/[Fe} \mathrm{II]} \mathrm{was} \mathrm{found.} \mathrm{This} \mathrm{could} \mathrm{be} \mathrm{caused} \mathrm{by} \mathrm{the} \mathrm{faint-}$ ness of the spectra, noting the high extinction to the object, and hence the high noise level. There may be a some evidence of He I $7001 \AA$ and N I 8680, 8703, $8711 \AA$ emission. However, due again to noise within the spectra and fringing effects at these longer wavelengths, it is difficult to calculate their 
Table 3. 2MASS apparent and absolute magnitudes of V1721 Aql progenitor (candidate), colours, and de-reddened colours of the near-IR source located at the position of the nova.

\begin{tabular}{cccccc}
\hline \hline Filter & Apparent magnitude & Absolute magnitude & Colour & Value & De-reddened value \\
\hline$J$ & $16.6 \pm 0.2$ & $1.8 \pm 0.6$ & $J-K_{\mathrm{s}}$ & $2.0 \pm 0.2$ & $0.1 \pm 0.2$ \\
$H$ & $15.5 \pm 0.1$ & $1.7 \pm 0.6$ & $J-H$ & $1.2 \pm 0.2$ & $0.0 \pm 0.2$ \\
$K_{\mathrm{s}}$ & $14.7 \pm 0.1$ & $1.7 \pm 0.6$ & $H-K_{\mathrm{s}}$ & $0.8 \pm 0.2$ & $0.1 \pm 0.2$ \\
\hline
\end{tabular}

Notes. The extinction towards the nova has been taken as $A_{\mathrm{V}}=11.6$, and its distance as $2.2 \mathrm{kpc}$.

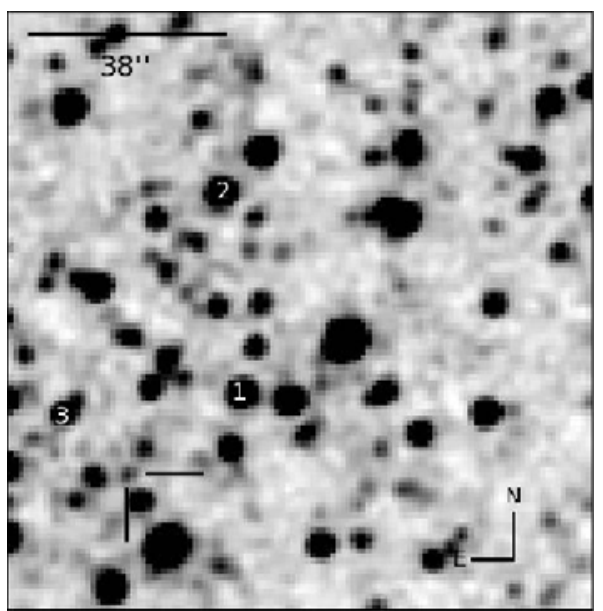

(a)

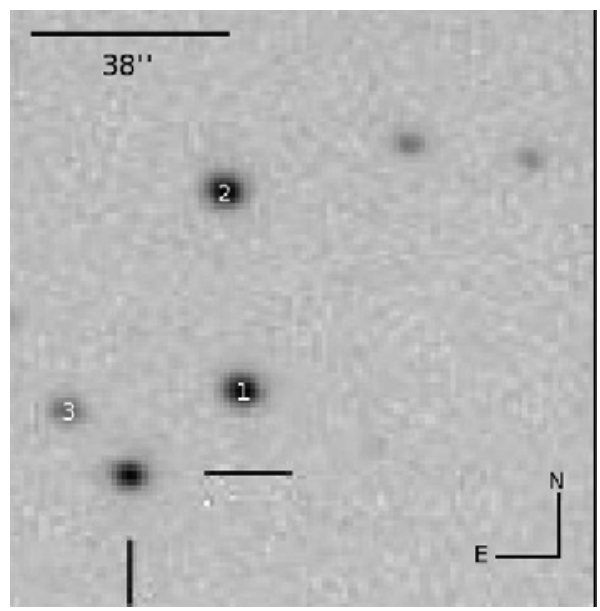

(b)
Fig. 7. a) $2 \mathrm{MASS} K_{\mathrm{S}}$ band pre-outburst image. The nova is found within the centre of the black markers. b) Unfiltered discovery image taken by K. Itagka on the 22nd of September at the Itagaki Astronomical Observatory. The nova is found within the centre of the black markers. significance. Exact spectral classification of the object according to the Williams (1992) system therefore remains elusive.

\subsection{Pre-outburst identification}

Pre-outburst images of a source at the location of V1721 Aql are found within the 2 MASS catalogue ${ }^{6}$, with near-IR co-ordinates given as $\alpha=19^{\mathrm{h}} 06^{\mathrm{m}} 28^{\mathrm{s}} .60, \delta=+07^{\circ} 06^{\prime} 44^{\prime \prime}$ '46; J2000. Observed 2MASS apparent magnitudes and colours of the near-IR source located at the position of the nova can be found in Table 3 . This table also contains de-reddened colours using the extinction value $A_{\mathrm{V}}=11.6 \pm 0.2$.

The V1721 Aql discovery image ${ }^{7}$ and 2MASS $K_{\mathrm{s}}$ image were aligned and compared via IRAF packages. Based on the stellar density within the 2MASS $K_{\mathrm{S}}$ pre-outburst image the probability of a chance alignment at least as close as that found between the nova and the 2MASS object is less than $1 \%$. The archival 2MASS $K_{\mathrm{s}}$ band image is presented in Fig. 7a and the discovery image presented in Fig. $7 b$.

\subsection{The nature of the secondary}

There are several factors that contribute to the observed near-IR colours of a nova system in quiescence, (i) the spectral type of the secondary and evolutionary phase; (ii) the rate of mass transfer $\dot{M}$; (iii) the extinction $A_{\mathrm{V}}$; (iv) the accretion disc and its inclination $i$; and (v) the mass of the primary. In $\mathrm{CNe}$ one would expect that the effect of the emission from the WD on the near-IR colours to be negligible, and the accretion disc to only provide a significant contribution to the emission when $i \lesssim 30^{\circ}$, where an

\footnotetext{
6 Available from

http://irsa.ipac.caltech.edu/applications/Gator/

7 Available from

http://www . astroalert.su/2008/09/24/nova-aql-2008
}

angle of $i=90^{\circ}$ is defined as an edge-on accretion disc (Weight et al. 1994). The location of a quiescent nova on a near-IR twocolour diagram $\left(H-K_{\mathrm{s}}, J-H\right)$ is therefore an important determinant of the nature of the secondary star in the system.

The near-IR apparent colours of the V1721 Aql nova system in quiescence are shown in Fig. 8. The system's colours occupy a region which contains the RN V745 Sco, which has a giant secondary with an M5+ III spectral type, and the suspected recurrent V1172 Sgr (Weight et al. 1994), which is also thought to contain a giant secondary. The extinction of these novae however, is much lower (for V745 Sco $A_{\mathrm{V}}=3.1 \pm 0.6$; Schaefer 2010) than that of V1721 Aql. Nova V1721 Aql's occupancy of this region is merely coincidental and does not indicate that it is a RN-like system. Nova Aql's de-reddening vector is indicated with a red line, the arrow head on this line represents an extinction value of $A_{\mathrm{V}}=11.6$, the surrounding red region represents the error circle of the corrected colours. The de-reddened quiescent near-IR colours of the V1721 Aql nova system lie within a region occupied by many quiescent $\mathrm{CNe}$. Assuming that the near-IR emission of the nova system is dominated by the secondary, Fig. 8 indicates that its spectral type is that of a late F-G (possibly K) main sequence star. However, the inclination of the accretion disc must be taken into account and if it is less than $30^{\circ}$ (approaching face-on to the observer) then its contribution to the near-IR colours would be to cause a significant blue-wards offset.

The line profiles observed in the nova spectra, and resultant high velocities, would suggest that the inclination of the disc within the binary system is low (face-on). The blue and red peaks seen within the $\mathrm{H} \alpha$ and $\mathrm{O}$ I structures would therefore be the result of material ejected along the poles towards and away from the observer (observations and shaping models predict that the minor axis of a remnant lies in the disc plane; Slavin et al. 1995; Porter et al. 1998). This inclination however, would 


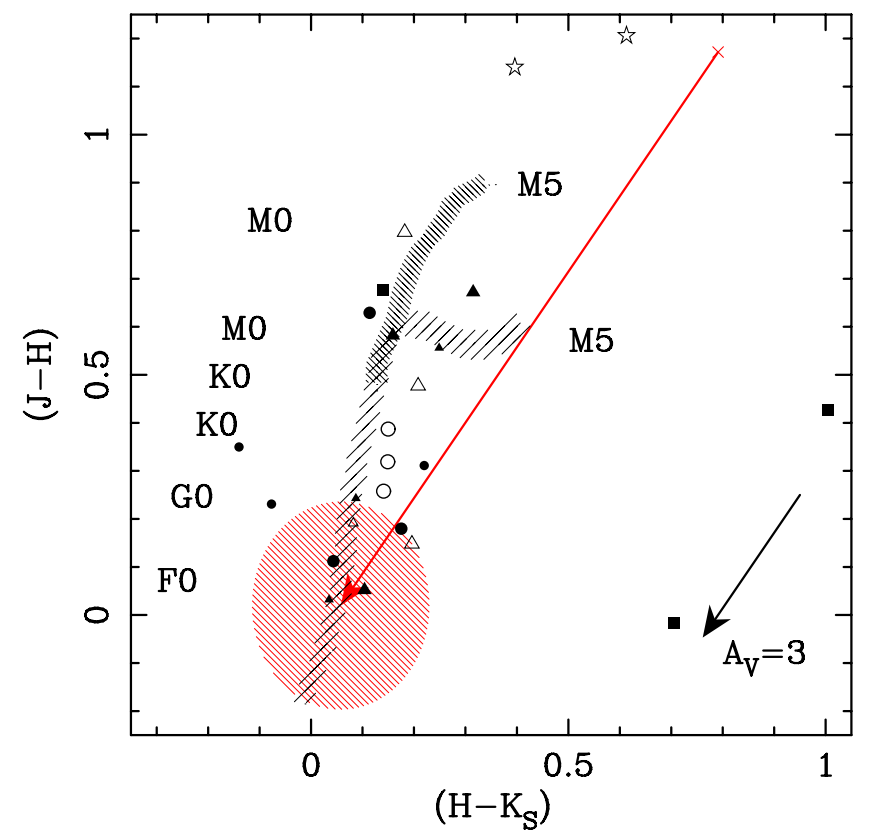

Fig. 8. Near-IR colour-colour diagram of quiescent classical nova systems reproduced from Figs. 4 and 7 in Hoard et al. (2002) using Table 1 of their data. The figure is adjusted to include the quiescent 2MASS colours of the nova V1721 Aql system. The light crosshatched area represents near-IR colours of main sequence stars with the denser cross-hatched area representing the giant branch (see references within Hoard et al. 2002). The black points show individual nova systems and are coded according to the time since outburst, $\tau$, as follows; filled squares: $\tau<25$ years; filled triangles: $\tau=25-50$ years; open triangles: $\tau=50-75$ years: filled circles: $\tau=75-100$ years; open circles: $\tau>100$ years. The star-shaped points are the recurrent nova systems. The nova systems presented here have not been corrected for extinction as in most cases the reddening is not accurately known, but it is assumed to be small to negligible in the near-IR for most Galactic novae systems. The large points for individual nova systems have $1 \sigma$ uncertainties of $\leq 0.1 \mathrm{mag}$, smaller points have $1 \sigma$ uncertainties of $>0.1 \mathrm{mag}$. The red cross represents the observed quiescent near-IR colours of the V1721 Aql nova system. The red line indicates the system's reddening vector with the arrowhead indicating its near-IR colours once corrected for an extinction of $A_{\mathrm{V}}=11.6$. The region enclosed by the red cross-hatching indicates all colours the nova system could possess within the error circle of $A_{\mathrm{V}}=11.6 \pm 0.2$. A de-reddening vector corresponding to $A_{\mathrm{V}}=3$ is also shown.

mean that the contribution by the disc to the near-IR colours is significant. Taking this blue contribution into account shifts the near-IR system colours along the main sequence and into the sub-giant region. Based on the speed and luminosity of the nova, the object may therefore be thought of as a U Sco type RN system. Comparisons at quiescence between the absolute $J$ band magnitudes and $H-K_{\mathrm{S}}$ colours of V1721 Aql (see Table 3), $\mathrm{U} \operatorname{Sco}\left(M_{J}=1.3 \pm 0.4, H-K_{\mathrm{S}}=0.0 \pm 0.1^{8}\right)$, and V2491 Cyg $\left(M_{J}=1.0 \pm 0.3\right.$, Darnley et al. Darnley et al. (2011), a suspected recurrent nova belonging to the U Sco class) support this argument as they all possess similar absolute $J$ magnitudes and occupy the same region of space in an equivalent colour magnitude diagram around the sub-giant branch. The probability of a red giant as the secondary can also be ruled out as the $J$ band absolute magnitude of the system would have to be approximately five magnitudes brighter.

\footnotetext{
8 Photometry taken from (Hanes 1985), distance and extinction taken from (Schaefer 2010).
}

Given the speed of decline of the nova, work by Slavin et al. (1995) would suggest that the axis ratio (ratio of semi-major to semi-minor axis) of Nova V1721 Aql's ejected shell is low $(\approx 1)$. The nova ejecta may therefore be modelled by an approximately spherical shell with discreet randomly distributed knots of brighter emission. We attempted to model such a nova system by calculating the expected emission line profiles from models of the ejecta distribution and comparing them to observed profiles, specifically $\mathrm{H} \alpha$. In order to do this we used XS5, a morphological and kinematical modelling programme (Harman et al. 2003) for producing 3D representations of astrophysical shells, synthetic images, and spectra. This program allows the user to generate a geometrical shape, such as an ellipsoid or an hourglass, which can be rotated and inclined. By adjusting additional parameters, such as the major and minor axis lengths, the FWHM of line profiles from the shell, the polar axis emission gradient, and the expansion velocity, the output emission line profile can be altered until a match with observations is achieved. Models of the nova ejecta with axis ratios between 1.0 and 2.0 (at 0.1 increments) were created. The results of this program are presented in Figs. 9 and 10. Figure 9c presents two modelled spectra compared to the observed $\mathrm{H} \alpha$ structure. The red spectrum is that of a spherical shell, axis ratio of 1 and the blue spectrum is of an ellipsoidal-like shell with an axis ratio of 1.4. Both shells are smooth with uniform emission, and the inclination of the system is such that the central accretion disc is face-on. Figure 10c illustrates the results from the same two structures with the same inclination, but this time there is a slight emission enhancement in the equatorial region. From these modelled spectra it would seem that an ellipsoidal-like morphology may actually be more suited to the V1721 Aql ejecta, however we have far too little information to make a strong argument for this. We note that this higher axis ratio is contradictory to expectations in Slavin et al. (1995). However, recent work on the 2010 outburst of U Sco by Drake \& Orlando (2010) has indicated that nova ejecta can be significantly shaped by circumbinary gas and/or a high accretion disc gas density. We have also been unable to reproduce the stronger red peak of the $\mathrm{H} \alpha$ emission line profile. This could possibly be due to clumps in the ejecta, but more detailed data and modelling are needed to explore this further.

\section{Discussion and conclusions}

The results presented in this paper indicate that V1721 Aql is a very fast nova ( $t_{2} \sim 6$ days) and very luminous $\left(M_{\mathrm{V}}=\right.$ $-9.4 \pm 0.5)$. The extinction of the object is high, $A_{\mathrm{V}}=11.6 \pm 0.2$ as the nova is very close to the Galactic plane. Based on the value of $A_{\mathrm{V}}$, the distance to the nova is estimated to be $2.2 \pm 0.6 \mathrm{kpc}$.

Pre-outburst near-IR colours of the nova have been compared to other novae in quiescence (all post-outburst) and the near-IR colours of main sequence and giant stars. The results indicate that, when de-reddened, the nova occupies a region of the colour-colour phase-space in which most $\mathrm{CNe}$ are found and appears to have a late (F-M) main sequence secondary or a sub-giant. However, we cannot rule out the possibility of V1721 Aql being a RN, only that it does not appear to contain a giant secondary and can therefore not belong to the RS Oph or $\mathrm{T} \mathrm{CrB}$ class of recurrent. The U Sco class of RNe however, consists of an evolved main sequence or sub-giant secondary, much like $\mathrm{CNe}$, and like V1721 Aql, such novae are very fast. Similarities in absolute $J$ band magnitudes at quiescence between V1721 Aql, U Sco, and V2491 Cyg (suspected U Sco member) also indicate that this object may be a U Sco type RN. 


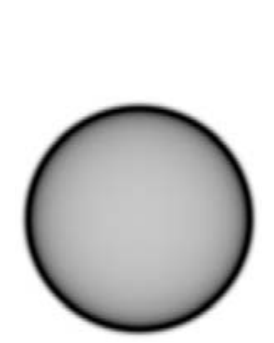

(a)

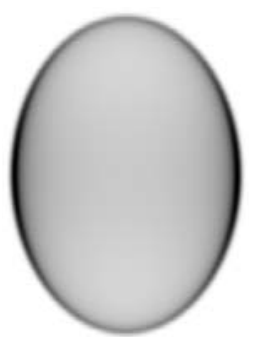

(b)

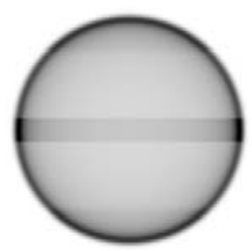

(a)

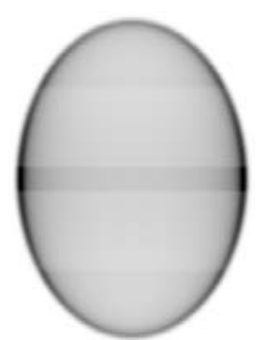

(b)

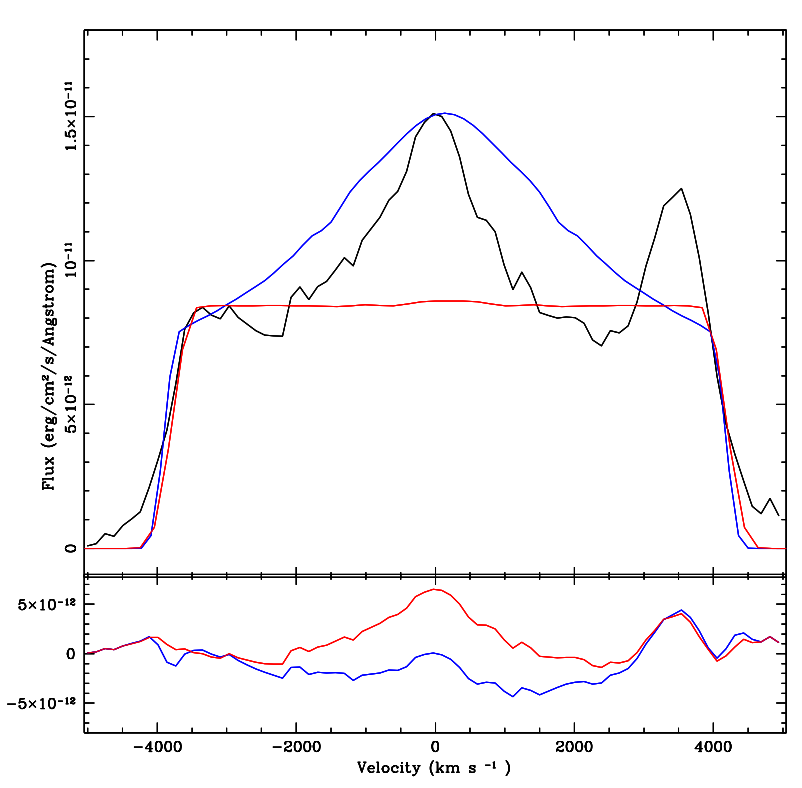

(c)

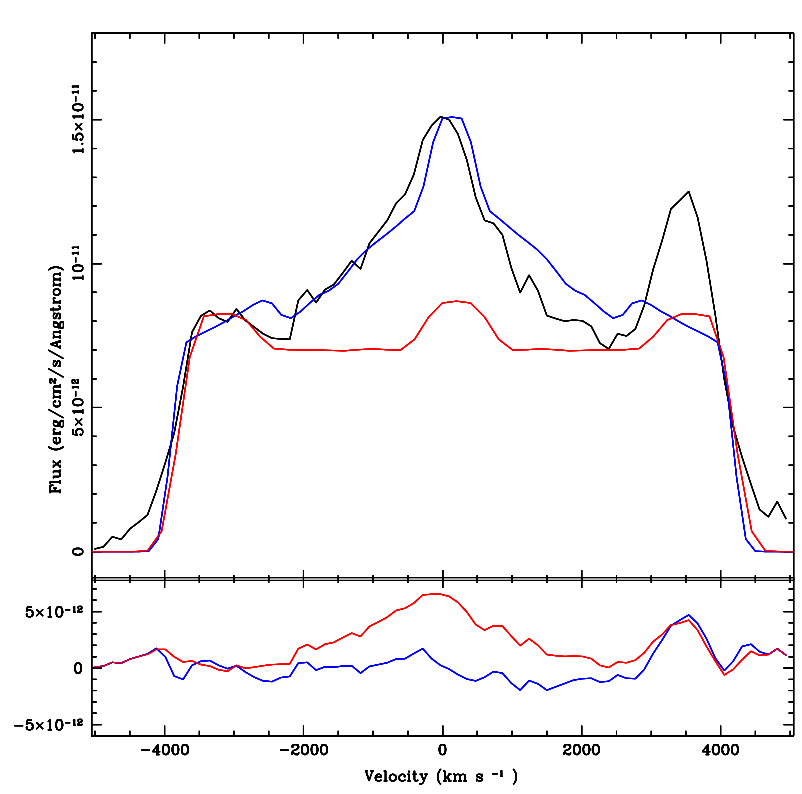

(c)
Fig. 9. a) Plan view of modelled nova ejecta with an axis ratio of 1 , the shell is smooth with uniform emission. b) Plan view of modelled nova ejecta with an axis ratio of 1.4, inclination of the system is such that the accretion disc is face-on to the observer, the shell is smooth with uniform emission. c) Relative velocity diagram of the observed $\mathrm{H} \alpha$ (black) structure and the two modelled systems created in XS5. The red line represents the system with an axis ratio of 1 , the blue line represents the system with an axis ratio of 1.4. The difference between the modelled and observed line profiles is given in the lower part of the diagram.

Post-outburst spectra of the V1721 Aql revealed boxy structures around $\mathrm{H} \alpha$, O I 7773, and O I $8446 \AA$. We note that similar complex $\mathrm{H} \alpha$ profiles have been observed in other fast novae such as the 1999 outburst of U Sco (Iijima 2002), and the 2009 nova V2672 Oph (Munari et al. 2011a), a suspected U Sco type object. Examination indicates that the features in V1721 Aql are not contaminated significantly by other emission lines. The structure of the line emission would suggest that material is being ejected from the poles of the nova shell moving towards and away from the observer, leading to the blue and red wing emission seen. This would indicate that the disc of the binary is faceon, an argument which is also supported when considering the high observed velocity of the system. If the accretion disc were edge-on, concealed velocities would be greater than those observed, this is unlikely. A face-on accretion disc is also more likely when considering the physical reasons for Gaussian 3 of Fig. 3. The Gaussian may represent a narrow core of $\mathrm{H} \alpha$ emission, in which case we are seeing $\mathrm{H}$ recombination emission both from the expanding ejecta, which gives rise to the broad overall
Fig. 10. a) Plan view of modelled nova ejecta with an axis ratio of 1 , inclination of the system is such that the accretion disc is face-on to the observer, the shell is smooth with a slight emission enhancement within the equatorial region. b) Plan view of modelled nova ejecta with an axis ratio of 1.4 , inclination of the system is such that the accretion disc is face-on to the observer, the shell is smooth with a slight emission enhancement within the equatorial region. c) Relative velocity diagram of the observed $\mathrm{H} \alpha$ (black) structure and the two modelled systems created in XS5. The red line represents the system with an axis ratio of 1 , the blue line represents the system with an axis ratio of 1.4. The difference between the modelled and observed line profiles is given in the lower part of the diagram.

emission, and emission from a reestablished accretion disc, or possibly even a disc that was never completely disrupted.

With a face-on accretion disc the hotter inner region of the disc is exposed possibly giving a significant blue contribution to the near-IR colours of the nova and thus seriously affecting previous spectral classification of the secondary. The speed of decline of the nova also suggest that the nova shell itself has a low axis ratio so that it is almost spherical. Basic models generated by XS5 to reproduce the $\mathrm{H} \alpha$ line profile, however, produce a best fit when using an ellipsoidal-like shell with an axis ratio 1.4. The departure from a spherical shell is most likely due to the nature of the explosion environment (circumbinary material and/or high density disc gas; Drake \& Orlando 2010). The model shell produced is smooth with a slight emission enhancement within the equatorial region, implying a face-on central accretion disc.

Relative velocity shifts found via spectral fitting of the $\mathrm{H} \alpha$ and $\mathrm{O}$ I emission are comparable to those presented in Helton et al. (2008) and we estimate an ejecta expansion 
velocity of $V_{\exp }=3400 \pm 200 \mathrm{~km} \mathrm{~s}^{-1}$ along the line of sight. This $V_{\exp }$ is, perhaps, more consistent with that of a fast classical nova system rather than fast recurrent novae, which tend to have slightly higher expansion velocities of $V_{\exp } \gtrsim 4000 \mathrm{~km} \mathrm{~s}^{-1}$ (Anupama 2008).

There is no evidence of emission blue-wards of the $\mathrm{H} \alpha$ structure. This is likely due to the high extinction towards the nova. An alternative explanation is that the nova is of the Fe II class and at this stage the shell is still optically thick. However, no dominant lines of Fe II or [Fe II] are present within the spectra. These lines may not have developed yet or may have been lost within the noise of the spectra. Therefore, an Fe II classification can not be ruled out. Another complication for this hypothesis is that Fe II novae tend to be slower than V1721 Aql.

The spectra show no conclusive evidence of $\mathrm{He}$ and $\mathrm{N}$ emission. This may also be due to the low signal to noise level within the spectra and the high extinction. No absorption features are seen in the spectra. This is unusual as we would expect to see absorption lines within the optically thick expansion stage.

In conclusion the precise nova sub-class of this object remains elusive, and the results of this work suggest two possibilities. The first is that this is a highly energetic luminous and fast classical nova with a main sequence secondary of spectral type F-M, and that any Fe II lines that may have been observable within the nova spectra have simply been extinguished. The second possibility is that this is a U Sco type RN and that evidence of $\mathrm{He} / \mathrm{N}$ within the spectra is lost due to the high extinction. The latter scenario may prove itself within the next few decades and therefore this object is one that merits continued monitoring for future outbursts.

Acknowledgements. This publication makes use of data products from the Two Micron All Sky Survey, which is a joint project of the University of Massachusetts and the Infra-red Processing and Analysis Center/California Institute of Technology, funded by the National Aeronautics and Space Administration and the National Science Foundation. We would like to thank Stephen Smartt and Rubina Kotak of Queen's University Belfast for pointing out this very interesting nova and providing us with further information. We would also like to thank an anonymous referee for detailed and thoughtful comments that have helped improve the paper. R. Hounsell is supported by a Ph.D. studentship from the Science and Technology Facilities Council of the UK.

\section{References}

Anupama, G. C. 2008, in RS Ophiuchi (2006) and the Recurrent Nova Phenomenon, ed. A. Evans, M. F. Bode, T. J. O’Brien, \& M. J. Darnley, ASP Conf. Ser., 401, 31

Bode, M. F. 2010, Astron. Nachr., 331, 160

Bode, M. F., \& Evans, A. 2008, Classical Novae, 2nd ed., ed. M. F. Bode, \& A. Evans (Cambridge: Cambridge University Press), Cambridge Astrophys. Ser., 43

Darnley, M. J., et al. 2011, A\&A, 530, A70

della Valle, M., Bianchini, A., Livio, M., \& Orio, M. 1992, A\&A, 266, 232

Downes, R. A., \& Duerbeck, H. W. 2000, AJ, 120, 2007

Drake, J. J., \& Orlando, S. 2010, ApJ, 720, L195

Duerbeck, H. W. 1990, in Physics of Classical Novae, IAU Colloq., 122, ed. A. Cassatella, \& R. Viotti (Berlin: Springer Verlag), Lecture Notes in Physics, 369,34

Hanes, D. A. 1985, MNRAS, 213, 443

Harman, D. J., Bryce, M., O’Brien, T. J., \& Meaburn, J. 2003, in Planetary Nebulae: Their Evolution and Role in the Universe, ed. S. Kwok, M. Dopita, \& R. Sutherland, IAU Symp., 209, 531

Helton, L. A., Woodward, C. E., Vanlandingham, K., \& Schwarz, G. J. 2008, IAU Circ., 8989, 2

Hoard, D. W., Wachter, S., Clark, L. L., \& Bowers, T. P. 2002, ApJ, 565, 511

Hounsell, R., Bode, M. F., Hick, P. P., et al. 2010, ApJ, 724, 480

Iijima, T. 2002, A\&A, 387, 1013

McLaughlin, D. B. 1945, PASP, 57, 69

Munari, U., Ribeiro, V. A. R. M., Bode, M. F., \& Saguner, T. 2011a, MNRAS, 410,525

Munari, U., Siviero, A., Dallaporta, S., et al. 2011b, New A, 16, 209

Payne-Gaposchkin, C. 1957, The galactic novae, ed. C. Payne-Gaposchkin (Amsterdam: North-Holland Pub. Co.; New York: Interscience Publishers)

Porter, J. M., O’Brien, T. J., \& Bode, M. F. 1998, MNRAS, 296, 943

Prialnik, D., \& Kovetz, A. 1995, ApJ, 445, 789

Rowles, J., \& Froebrich, D. 2009, MNRAS, 395, 1640

Schaefer, B. E. 2010, ApJS, 187, 275

Shara, M. M. 1981, ApJ, 243, 268

Skrutskie, M. F., Beichman, C., Capps, R., et al. 1995, in BAAS, 27, 1392

Slavin, A. J., O’Brien, T. J., \& Dunlop, J. S. 1995, MNRAS, 276, 353

Starrfield, S., Iliadis, C., \& Hix, W. R. 2008, in Classical Novae, 2nd ed., ed. M. F. Bode, \& A. Evans (Cambridge: Cambridge University Press), Cambridge Astrophys. Ser., 43, 77

Warner, B. 2008, in Classical Novae, 2nd ed., ed. M. F. Bode, \& A. Evans (Cambridge: Cambridge University Press), Cambridge Astrophys. Ser., 43, 16

Weight, A., Evans, A., Naylor, T., Wood, J. H., \& Bode, M. F. 1994, MNRAS, 266, 761

Williams, R. E. 1992, AJ, 104, 725

Yamaoka, H., Itagaki, K., Nakano, S., et al. 2008, IAU Circ., 8989 